\title{
Impact of Protocol of Care of Patients Undergoing Urinary Catheterization on Nurses' practice
}

\author{
Prof. Labiba Abd Elkader Mohamed, Prof. Dr. Mamdouh Mohamad AlMezaien , \\ Ass. Prof. Amal Bakr Abo El-Ata, and Amira Tag Mohamed Abo-EI Nasser \\ Prof of Medical Surgical Nursing ～, Faculty of Nursing, Cairo University, \\ Professor of vascular surgery, Faculty of Medicine, Suez, Canal University, Assistant \\ Professor of Medical - Surgical Nursing, Faculty of Nursing - Port Said University, \\ Clinical Instructor, Al-Azhar University, Egypt.
}

\begin{abstract}
Background: Urinary catheterization is an invasive procedure carried out by nurses as part of the routine of their care practice. Hence, professional must have knowledge related to the management of urinary catheterization. Aim of the study was to evaluate the impact of protocol of care of patients undergoing urinary catheterization on nurses' practice. Subject and Method: A quasi- experimental research design was used to conduct this study. The study was carried out at Intensive Care Unit, Urology Department and Department of Internal Medicine at Al-Azhar University hospital in Damietta City to test the hypotheses that positive changes in nurses' practice after implementing the protocol of care for patients undergoing urinary catheterization. A convenient sample of 50 nurses was included. One tool was used for data collections, part (1) Structured Interview Questionnaire tool that includes demographic data part (2) Observational Checklist to evaluate nurses' practice in relation to care of patient patients undergoing urinary catheterization Results demonstrated that there were statistically significant improvements in the total score of nurses' practice regarding care of patients undergoing urinary catheterization throughout the protocol intervention. Recommendations: The study recommended that providing ongoing inservice education for nurses to update their practice related to care of patients undergoing urinary catheterization and catheter-associated problems.
\end{abstract}




\section{INTRODUCTION}

The indwelling urinary catheter (IUC) is a widely utilized device in modern hospital environments, yet they are not always used appropriately in hospital settings and can result in prolonged use and improper management, increasing risk of infections and length of stay. Nurses are the primary champions to promote preventative measures, provide patient education, and evaluate evidence-based practice (EBP) strategies to decrease catheter associated urinary tract infections (CAUTIs) (Gordon, 2015).

Nurses are able to perform the best possible IUC utilization assessment to patients, standardizing the health care quality. The culture of evidence-based practice can be promoted within the organization, motivating nurses and other clinical staff to pursue evidence-based practice in their scope of work and compete with the rapid clinical advances and information explosion. Furthermore, the new protocol may also strengthen the concept of patient safety and hospital acquired infection (HAI) prevention (Pui, 2011).Nurses are responsible for placing and maintaining the urinary catheter system; including cleaning and other tasks in addition nurses play a vital role in the diagnosis of catheter associated urinary tract infection (Yoon et al., 2013).

Among catheterized patients, nurses are often the first to notice a clinical change or technical problem. Routine care of the catheter is often delegated to non-licensed personnel. Inadequate placement and care from the beginning may contribute to increased infection rates. (Yoon et al., 2013). Despite being considered a common procedure, IUC it is associating with complications which require efforts from nurses to be controlled. Currently, there is an absence of standardization of the urinary catheterization technique in different health institutions so the Nursing professionals must have knowledge related to the management of an indwelling urinary catheter, complications caused by an indwelling urinary catheter and ways to prevent and manage these complications (Altun and Karakoc, 2010 and Ercole et al., 2013).

Focusing on nursing-driven interventions to improve the nursing care of IUCs was found to positively impact CAUTI rates. Reeducating on the importance of a 
perceived "basic" skill and infusing best evidence into current practice were important to raise awareness of simple interventions that positively impacted patient outcomes. Standardizing the education, training, and orientation of new nursing staff on proper urinary catheter placement and care can have an impact on patient outcomes (Oman et al., 2011).Therefore; the aim of the current study is to evaluate the impact of protocol of care of patients undergoing urinary catheterization on nurses' performance

\section{AIM OF STUDY:}

The aim of this study was to; Evaluate the impact of protocol of care of patients undergoing urinary catheterization on nurses' practice.

\section{Research Hypotheses:}

To fulfill the aim of this study the following hypothesis was formulated:

- There will be positive changes in nurses' practice after implementing the protocol of care for patients undergoing urinary catheterization.

\section{MATERIALS AND METHOD:}

\section{Research Design:}

A quasi-experimental study design was used for the conduction of this study.

\section{Setting:}

This study was conducted at Intensive Care Unit, Urology Department and Department of Internal Medicine at Al-Azhar University hospital in Damietta city.

\section{Subjects:}

A convenient sample of 50 nurses working with patients undergoing urinary catheterization at Intensive Care Unit, Urology Department and Department of Internal Medicine, at Al-Azhar University hospital in Damietta city.

\section{Exclusion criteria:}

- Student nurses of any educational level.

- Nurses who refuse to participate in the study 


\section{Tools of data collection:}

Tools used in this study were: one tool was used in this study

\section{Part (I): Structured interview Questionnaire:}

It was developed by the researcher to assess items related to socio- demographic characteristics and work related data of the studied nurses such as age, gender, marital status, working unit, level of education, years of experience and attending training program related to urinary catheter.

\section{Part (П): " Observational Checklist":}

It was developed by the researcher, after review of recent related literature to evaluate nurses' practice in relation to care of patient patients undergoing urinary catheterization. It consisted of 87 steps

\section{Scoring system:}

As regard, studied nurse's practice each step checked answer was scored one for (done) and checked (not done) was scored zero. The total nurse's practice were summed up then converted to percent. Total nurses practices were calculated as the following: All values $\geq 75 \%$ were considered satisfactory While values $<75 \%$ were considered unsatisfactory

\section{Assessment phase:}

In this phase after finalization of the tools, the researcher assessed nurses' learning needs using Tool was designed to assess nurse's practice, as each nurse was observed by the researcher during her actual clinical practice. The nurse was evaluated by using observational checklist (Tool). Moreover, the researcher assessed available place, time, equipment, supplies, and instructional materials for conduction of the protocol of care.

\section{The protocol of care development phase}

The protocol of care was developed based on the identified needs and demands of nurses gathered in assessment phase and review of related literature. This phase included the following;

\section{C.Setting objectives:}

The aim of protocol was to improve nurses practice related to care of patients undergoing urinary catheterization though: 
Improve nurses' practice related to care of patients undergoing urinary catheterization

\section{Preparation of the content:}

Content covered all areas about caring of patients undergoing urinary catheterization was prepared which included the following: preparation during urinary catheter insertion, nursing care for urinary catheter management ,emptying of drainage bag and removing of urinary catheter.

In this phase, the researcher designed a plan for a protocol of care implementation

\section{Implementation phase:}

After official permission was taken from the concerned study setting. The researcher took the list of nurses who met the inclusion criteria. The participated nurses were divided into 10 groups, each consisted of five nurses. Each group was attended at conference room separately during morning and afternoon shift. The purpose and aim of the study was explained, then the researcher collect data about demographic characteristics using tool (I). This session is considered as introductory session.

At the beginning of each session, pretest related to the session content was provided to participants, followed by hands out. During the session, the researcher teach content in a clear, simple language using lectures, illustrative pictures and discussion giving feedback using positive verbal words.

At the end of each session the researcher, close the session by summary for the main points. Posttest was at the end of the $4^{\text {th }}$ session using toll II.

\section{Evaluation phase:}

The protocol of care was evaluated three times using tool II. Tool II used to evaluate the studied nurses .Evaluation was done three times, first time: immediately after protocol implementation, second time after three Months, and third time: after six month.

\section{Ethical Consideration:}

Explain the aim of the study to the directors of Intensive Care Unit, Department of Urology and Internal Medicine to take their permission to start this study .Oral consent 
was taken from the study subjects after explaining the aims and nature of the study to them, and they were assured that the information collected would be treated confidentially and used for the research purpose only, and they have the right to withdraw from the study at any time.

\section{Statistical Design:}

The collected data organized, tabulated and statistically analyzed using statistical package for social science (SPSS) version 16 for windows, running on IBM compatible computer. Qualitative data (categorical data) were expressed as relative frequency (number) and percent distribution, and for comparison between groups, the Chi square $\left(\mathrm{X}^{2}\right)$ or Mann-Whitney test $(\mathrm{Z})$ was calculated. Quantitative data were expressed as mean $\pm \mathrm{SD}$, and for comparison between two means, the student ( $\mathrm{t}$ ) test was calculated. For interpretation of results, the $p$ value $\leq 0.05$ was considered significant.

\section{RESULTS:}

Table (1): shows the demographic and work related data of studied nurses. It revealed that (96\%) of the studied nurses were females and (90\%) their age from 21 to 36 years. There were $46 \%$ of studied nurses had technical nursing institute diploma , while only (16\%) had nursing bachelor; and (48\%) had less than five years of experience and $(50.0 \%)$ working in intensive care unit (ICU) and (30\%) work in urology unit. All studied nurses (100\%) have not any previous training course about urinary catheterization.

Table (2): Shows that Percentage distribution of studied nurses' satisfactory practice related to urinary catheterization procedure throughout the protocol intervention and revealed that before intervention (94\%) have satisfactory practice score, immediate after (100\%), 3 month after (100\%) and 6 month after intervention (100\%) have total satisfactory practice score Moreover, there were statistically significant differences $(X 2=9.13, \mathrm{p}=0.028)$ about urinary catheterization procedures while, there were no statistically significant differences regarding applying sterile drape $(\mathrm{X} 2=2.02, \mathrm{p}=0.56)$.

Table (3): Shows Percentage distribution of studied nurses' satisfactory practice related to urinary catheter management throughout the protocol intervention $(\mathrm{No}=50)$ and revealed that before intervention (14\%) have satisfactory practice score, immediate after 
(32\%), 3 month after (28\%) and 6 month after intervention (28\%) have total satisfactory practice score but there were no statistically significant differences $(X 2=4.80, \mathrm{p}=0.18)$ about urinary catheter management. Moreover, there were statistically significant differences as regard hand washing before handling catheter $\left(X^{2}=34.09, \mathrm{p}<0.001\right)$, wear clean disposable gloves $\left(\mathrm{X}^{2}=81.8, \mathrm{p}<0.001\right)$ and prevent kinking of catheter $\left(\mathrm{X}^{2}=35.29, \mathrm{p}<0.001\right)$,

Table ( $₹$ ): shows that there were statically significant differences in the total score of nurses' practice regarding care of patient undergoing urinary catheterization throughout the protocol intervention between immediately posttest, pre protocol first follow up and pre protocol, second follow up, and pre protocol $(\mathrm{P}<0.001)$. There were no statistically significant differences between immediately posttest and first follow up $(\mathrm{P}=0.076)$. In addition, there was no statistical significant difference between first and second follow-up $(\mathrm{P}=0.68)$.

Table (5): Shows that there were no statistical significance differences between studied nurse's knowledge or practice and their age groups throughout the protocol intervention.

Table (21): Shows regarding knowledge there no statistically significant differences between studied nurses' knowledge and their experience throughout the protocol intervention except before the protocol $(\mathrm{F}=3.48, \mathrm{p}=0.039)$. Regarding practice there no statistically significant differences between studied nurses' practice and their experience throughout the protocol intervention except before the protocol $(F=3.50, p=0.038)$. 
Table (1): Distribution of the studied nurses according to their demographic characteristics and work related data $(\mathrm{No}=50)$

\begin{tabular}{|c|c|c|c|}
\hline \multicolumn{2}{|l|}{ Items } & $\mathbf{N}$ & $\%$ \\
\hline \multirow[t]{2}{*}{-Age } & Less than 21years & 5 & 10.0 \\
\hline & 21 to 36 years & 45 & 90.0 \\
\hline \multirow[t]{2}{*}{-Gender } & Male & 2 & 4.0 \\
\hline & Female & 48 & 96.0 \\
\hline \multirow[t]{4}{*}{-Marital state } & single & 9 & 18.0 \\
\hline & Married & 40 & 80.0 \\
\hline & Divorced & 1 & 2.0 \\
\hline & Widow & 0 & 0.0 \\
\hline \multirow[t]{3}{*}{-Education level } & $\begin{array}{ll}\text { Secondary school } \\
\text { diploma }\end{array}$ & 19 & 38.0 \\
\hline & $\begin{array}{l}\text { Technical nursing } \\
\text { institute diploma }\end{array}$ & 23 & 46.0 \\
\hline & Bch degree of nursing & 8 & 16.0 \\
\hline \multirow[t]{3}{*}{-Years of experience } & Less than 5 years & 24 & 48.0 \\
\hline & 5 to 10 years & 20 & 40.0 \\
\hline & More than 10 years & 6 & 12.0 \\
\hline \multirow[t]{3}{*}{-Work Department } & Urology & 15 & 30.0 \\
\hline & ICU & 25 & 50.0 \\
\hline & Internal medicine & 10 & 20.0 \\
\hline \multirow[t]{2}{*}{-Training course } & yes & 0 & 0.0 \\
\hline & No & 50 & 100.0 \\
\hline
\end{tabular}


Table (2): Percentage distribution of studied nurses' satisfactory practice related to urinary catheterization procedure throughout the protocol intervention $(\mathrm{No}=50)$

\begin{tabular}{|c|c|c|c|c|c|c|c|c|c|c|c|}
\hline \multirow{2}{*}{\multicolumn{2}{|c|}{ ITEMS }} & \multicolumn{2}{|c|}{$\begin{array}{c}\text { Before } \\
\text { intervention }\end{array}$} & \multicolumn{2}{|c|}{$\begin{array}{c}\text { Immediately } \\
\text { After }\end{array}$} & \multicolumn{2}{|c|}{3 Months After } & \multicolumn{2}{|c|}{6 Months After } & \multirow[t]{2}{*}{$\mathbf{X}^{2}$} & \multirow[t]{2}{*}{ P-value } \\
\hline & & N. & $\%$ & N. & $\%$ & N. & $\%$ & N. & $\%$ & & \\
\hline \multicolumn{2}{|c|}{ Picking up catheter with.. } & 50 & $100.0 \%$ & 50 & $100.0 \%$ & 50 & $100.0 \%$ & 50 & $100.0 \%$ & $\mathrm{a}$ & \\
\hline \multicolumn{2}{|c|}{ Lubricating the tip of.... } & 47 & $94.0 \%$ & 50 & $100.0 \%$ & 50 & $100.0 \%$ & 50 & $100.0 \%$ & 9.13 & $0.028 *$ \\
\hline \multicolumn{2}{|c|}{ Inserting catheter to... } & 50 & $100.0 \%$ & 50 & $100.0 \%$ & 50 & $100.0 \%$ & 50 & $100.0 \%$ & $\mathrm{a}$ & \\
\hline \multicolumn{2}{|c|}{ Advance catheter until... } & 50 & $100.0 \%$ & 50 & $100.0 \%$ & 50 & $100.0 \%$ & 50 & $100.0 \%$ & $\mathrm{a}$ & \\
\hline \multicolumn{2}{|c|}{ Advance the catheter another } & 50 & $100.0 \%$ & 50 & $100.0 \%$ & 50 & $100.0 \%$ & 50 & $100.0 \%$ & $\mathrm{a}$ & \\
\hline \multicolumn{2}{|c|}{ Don't force the catheter } & 50 & $100.0 \%$ & 50 & $100.0 \%$ & 50 & $100.0 \%$ & 50 & $100.0 \%$ & $\mathrm{a}$ & \\
\hline \multicolumn{2}{|c|}{$\begin{array}{l}\text { Asking patient to breathe } \\
\text { during insertion }\end{array}$} & 29 & $58.0 \%$ & 49 & $98.0 \%$ & 47 & $94.0 \%$ & 46 & $92.0 \%$ & 41.42 & $<0.001 * *$ \\
\hline \multicolumn{2}{|l|}{ Inflate balloon } & 50 & $100.0 \%$ & 50 & $100.0 \%$ & 50 & $100.0 \%$ & 50 & $100.0 \%$ & $\mathrm{a}$ & \\
\hline \multicolumn{2}{|c|}{ Testing the balloon } & 50 & $100.0 \%$ & 50 & $100.0 \%$ & 50 & $100.0 \%$ & 50 & $100.0 \%$ & $\mathrm{a}$ & \\
\hline \multicolumn{2}{|c|}{ Securing the catheter } & 5 & $10.0 \%$ & 33 & $66.0 \%$ & 34 & $68.0 \%$ & 34 & $68.0 \%$ & 9.53 & $<0.001 * *$ \\
\hline \multirow[t]{2}{*}{$\begin{array}{l}\text { Attach drainage } \\
\text { bag to }\end{array}$} & $\begin{array}{l}\text { Bed } \\
\text { frame }\end{array}$ & 30 & $60.0 \%$ & 33 & $66.0 \%$ & 29 & $58.0 \%$ & 28 & $56.0 \%$ & 1.16 & $0.76(\mathrm{NS})$ \\
\hline & Stand & 0 & $0.0 \%$ & 0 & $0.0 \%$ & 0 & $0.0 \%$ & 2 & $4.0 \%$ & 6.06 & $0.11(\mathrm{NS})$ \\
\hline \multicolumn{2}{|c|}{ Left bag free on the floor } & 20 & $40.0 \%$ & 17 & $34.0 \%$ & 21 & $42.0 \%$ & 20 & $40.0 \%$ & 0.75 & $0.86(\mathrm{NS})$ \\
\hline \multicolumn{2}{|c|}{ Discard contaminated equip. } & 50 & $100.0 \%$ & 50 & $100.0 \%$ & 50 & $100.0 \%$ & 50 & $100.0 \%$ & a & \\
\hline \multicolumn{2}{|l|}{ Remove gloves } & 50 & $100.0 \%$ & 50 & $100.0 \%$ & 50 & $100.0 \%$ & 50 & $100.0 \%$ & $\mathrm{a}$ & \\
\hline \multicolumn{2}{|l|}{ Hand washing } & 50 & $100.0 \%$ & 50 & $100.0 \%$ & 48 & $96.0 \%$ & 48 & $96.0 \%$ & 4.08 & $0.25(\mathrm{NS})$ \\
\hline \multicolumn{2}{|c|}{ Recording and reporting } & 50 & $100.0 \%$ & 50 & $100.0 \%$ & 50 & $100.0 \%$ & 50 & $100.0 \%$ & $\mathrm{a}$ & \\
\hline \multicolumn{2}{|l|}{ Total } & 47 & $94.0 \%$ & 50 & $100.0 \%$ & 50 & $100.0 \%$ & 50 & $100.0 \%$ & 9.13 & $0.028 *$ \\
\hline
\end{tabular}


Table (3): Percentage distribution of studied nurses' satisfactory practice related to urinary catheter management throughout the protocol intervention $(\mathrm{No}=50)$

\begin{tabular}{|c|c|c|c|c|c|c|c|c|c|c|}
\hline \multirow[t]{2}{*}{ ITEMS } & \multicolumn{2}{|c|}{$\begin{array}{l}\text { Before } \\
\text { intervention }\end{array}$} & \multicolumn{2}{|c|}{$\begin{array}{c}\text { Immediately } \\
\text { After }\end{array}$} & \multicolumn{2}{|c|}{3 months After } & \multicolumn{2}{|c|}{6 Months After } & \multirow[t]{2}{*}{$\mathbf{X}^{2}$} & \multirow[t]{2}{*}{ P-value } \\
\hline & N. & $\%$ & N. & $\%$ & $\mathbf{N}$. & $\%$ & $\mathbf{N}$. & $\%$ & & \\
\hline $\begin{array}{l}\text { Hand washing before UC } \\
\text { handling }\end{array}$ & 10 & $20.0 \%$ & 37 & $74.0 \%$ & 32 & $64.0 \%$ & 30 & $60.0 \%$ & 34.09 & $<0.001^{* *}$ \\
\hline $\begin{array}{l}\text { Hand washing after } \\
\text { handling }\end{array}$ & 50 & $100.0 \%$ & 50 & $100.0 \%$ & 50 & $100.0 \%$ & 50 & $100.0 \%$ & $\mathrm{a}$ & \\
\hline $\begin{array}{l}\text { Wear clean disposable } \\
\text { gloves }\end{array}$ & 26 & $52.0 \%$ & 50 & $100.0 \%$ & 50 & $100.0 \%$ & 50 & $100.0 \%$ & 81.8 & $<0.001 * *$ \\
\hline Antiseptic before emptying & 0 & $.0 \%$ & 1 & $2.0 \%$ & 0 & $.0 \%$ & 0 & $.0 \%$ & 3.01 & $0.38(\mathrm{NS})$ \\
\hline Antiseptic after emptying & 0 & $.0 \%$ & 1 & $2.0 \%$ & 0 & $.0 \%$ & 0 & $.0 \%$ & 3.01 & $0.38(\mathrm{NS})$ \\
\hline $\begin{array}{l}\text { Urine received in sterile } \\
\text { container }\end{array}$ & 0 & $.0 \%$ & 1 & $2.0 \%$ & 0 & $.0 \%$ & 1 & $2.0 \%$ & 2.02 & $0.56(\mathrm{NS})$ \\
\hline Assess urethral meatus & 34 & $68.0 \%$ & 36 & $72.0 \%$ & 35 & $70.0 \%$ & 34 & $68.0 \%$ & 0.25 & $0.96(\mathrm{NS})$ \\
\hline Clean perineum & 34 & $68.0 \%$ & 36 & $72.0 \%$ & 36 & $72.0 \%$ & 36 & $72.0 \%$ & 0.29 & $0.96(\mathrm{NS})$ \\
\hline Prevent kinking of tube & 16 & $32.0 \%$ & 40 & $80.0 \%$ & 40 & $80.0 \%$ & 36 & $72.0 \%$ & 35.29 & $<0.001 * *$ \\
\hline $\begin{array}{l}\text { Position uribag } \\
\text { appropriately }\end{array}$ & 29 & $58.0 \%$ & 36 & $72.0 \%$ & 35 & $70.0 \%$ & 35 & $70.0 \%$ & 2.80 & $0.42(\mathrm{NS})$ \\
\hline Avoid catheter clamping & 8 & $16.0 \%$ & 47 & $94.0 \%$ & 50 & $100.0 \%$ & 50 & $100.0 \%$ & 145.3 & $<0.001 * *$ \\
\hline Empty bag every 8 hours & 0 & $.0 \%$ & 0 & $0.0 \%$ & 0 & $.0 \%$ & 0 & $0.0 \%$ & $\mathrm{a}$ & \\
\hline Empty bag every 12 hours & 0 & $.0 \%$ & 0 & $0.0 \%$ & 0 & $.0 \%$ & 0 & $0.0 \%$ & $\mathrm{a}$ & \\
\hline Empty bag when needed & 50 & $100.0 \%$ & 50 & $100.0 \%$ & 50 & $100.0 \%$ & 50 & $100.0 \%$ & $\mathrm{a}$ & \\
\hline Check character of urine & 42 & $84.0 \%$ & 50 & $100 \%$ & 50 & $100.0 \%$ & 50 & $100.0 \%$ & 16.45 & $0.001 * *$ \\
\hline Encourage fluid intake & 46 & $92.0 \%$ & 50 & $100.0 \%$ & 50 & $100.0 \%$ & 50 & $100.0 \%$ & 12.24 & $0.007^{*}$ \\
\hline Record intake and output & 50 & $100.0 \%$ & 50 & $100.0 \%$ & 50 & $100.0 \%$ & 50 & $100.0 \%$ & $\mathrm{a}$ & \\
\hline $\begin{array}{l}\text { Check securing of uribag } \\
\text { system }\end{array}$ & 7 & $14.0 \%$ & 34 & $68.0 \%$ & 22 & $44.0 \%$ & 22 & $44.0 \%$ & 30.01 & $<0.001 * *$ \\
\hline $\begin{array}{l}\text { Keep uribag from touching } \\
\text { floor }\end{array}$ & 17 & $34.0 \%$ & 34 & $68.0 \%$ & 31 & $62.0 \%$ & 31 & $62.0 \%$ & 14.22 & $0.003^{*}$ \\
\hline Give patient health teaching & 12 & $24.0 \%$ & 32 & $64.0 \%$ & 32 & $64.0 \%$ & 32 & $64.0 \%$ & 24.15 & $<0.001 * *$ \\
\hline Total & 7 & $14.0 \%$ & 16 & $32.0 \%$ & 14 & $28.0 \%$ & 14 & $28.0 \%$ & 4.80 & $0.18(\mathrm{NS})$ \\
\hline
\end{tabular}


Table (4): Percentage distribution of the total score of nurse's practice regarding care of patients undergoing urinary catheterization throughout the protocol intervention $(\mathrm{No}=50)$

\begin{tabular}{|c|c|c|c|c|c|c|c|c|c|c|}
\hline & \multicolumn{2}{|c|}{$\begin{array}{l}\text { Before } \\
\text { intervention }\end{array}$} & \multicolumn{2}{|c|}{$\begin{array}{c}\text { Immediately } \\
\text { After }\end{array}$} & \multicolumn{2}{|c|}{$\begin{array}{l}3 \text { Months } \\
\text { After }\end{array}$} & \multicolumn{2}{|c|}{$\begin{array}{l}6 \text { Months } \\
\text { After }\end{array}$} & \multirow[t]{2}{*}{$\mathbf{X}^{2}$} & \multirow[t]{2}{*}{ P-V } \\
\hline & N. & $\%$ & N. & $\%$ & N. & $\%$ & N. & $\%$ & & \\
\hline Satisfied ( $\geq 75 \%)$ & 8 & $16.0 \%$ & 40 & $80.0 \%$ & 32 & $64.0 \%$ & 30 & $60.0 \%$ & \multirow{2}{*}{45.49} & \multirow{2}{*}{$<0.001^{* *}$} \\
\hline Unsatisfactory $(<75 \%)$ & 42 & $84.0 \%$ & 10 & $20.0 \%$ & 18 & $36.0 \%$ & 20 & $40.0 \%$ & & \\
\hline Mean \pm SD & \multicolumn{2}{|c|}{$64.95 \pm 7.38$} & \multicolumn{2}{|c|}{$80.38 \pm 5.65$} & \multicolumn{2}{|c|}{$78.32 \pm 6.18$} & \multicolumn{2}{|c|}{$77.47 \pm 6.57$} & $\mathrm{~F}=28.25$ & $<0.001 * *$ \\
\hline $\begin{array}{l}\text { Immediately Posttest- pre } \\
\text { protocol }\end{array}$ & \multicolumn{10}{|c|}{$\mathrm{Z}=6.37, \mathrm{p}<0.001^{*}$} \\
\hline F3-pre protocol & \multicolumn{10}{|c|}{$\mathrm{Z}=4.87, \mathrm{p}<0.001^{*}$} \\
\hline F6-Pre protocol & \multicolumn{10}{|c|}{$\mathrm{Z}=4.51, \mathrm{p}<0.001^{*}$} \\
\hline F3- Immediately Posttest & \multicolumn{10}{|c|}{$\mathrm{Z}=1.77, \mathrm{p}=0.076(\mathrm{NS})$} \\
\hline F6- Immediately Posttest & \multicolumn{10}{|c|}{$\mathrm{Z}=2.17, \mathrm{p}=0.03^{*}$} \\
\hline $\begin{array}{l}\text { F6-F3 } \\
\end{array}$ & \multicolumn{10}{|c|}{$\mathrm{Z}=0.41, \mathrm{p}=0.68(\mathrm{NS})$} \\
\hline
\end{tabular}

Table (5): Relation between mean score of studied nurses' total satisfactory knowledge and practice regarding care of patients undergoing UC throughout the protocol intervention according to according to their age $(\mathrm{No}=50)$

\begin{tabular}{|c|c|c|c|c|c|}
\hline & \multicolumn{2}{|l|}{ Age group } & \multirow[t]{2}{*}{ t- test } & \multirow[t]{2}{*}{ P-value } \\
\hline & & $\begin{array}{l}\leq 21 \text { years } \\
\text { Mean } \pm \text { SD }\end{array}$ & \begin{tabular}{|l|}
21 \\
Mean \pm SD
\end{tabular} & & \\
\hline \multirow{4}{*}{$\begin{array}{l}\text { Total satisfactory } \\
\text { Practice score }\end{array}$} & Before protocol & $64.02 \pm 2.53$ & $65.05 \pm 7.74$ & 0.29 & $0.76(\mathrm{NS})$ \\
\hline & $\begin{array}{l}\text { Immediately after } \\
\text { protocol }\end{array}$ & $83.22 \pm 8.57$ & $80.07 \pm 5.28$ & 1.18 & $0.24(\mathrm{NS})$ \\
\hline & 3 months after protocol & $80.57 \pm 8.45$ & $78.07 \pm 5.96$ & 0.85 & 0.39 (NS) \\
\hline & 6 months after protocol & $80.41 \pm 8.59$ & $77.15 \pm 6.34$ & 1.05 & $0.29(\mathrm{NS})$ \\
\hline
\end{tabular}


Table (6): Relation between mean score of studied nurses' total satisfactory knowledge and practice regarding care of patients undergoing UC throughout the protocol intervention according to their experience $(\mathrm{No}=50)$

\begin{tabular}{|c|c|c|c|c|c|c|}
\hline & & \multicolumn{3}{|l|}{ Years of experience } & \multirow[t]{3}{*}{$\mathbf{F}$} & \multirow[t]{3}{*}{$P$-value } \\
\hline & & Fewer Than5 Years & From 5 To 10 Years & More Than 10 Years & & \\
\hline & & Mean \pm S D & Mean \pm S D & Mean \pm S D & & \\
\hline \multirow{4}{*}{$\begin{array}{l}\text { Total } \\
\text { satisfactory } \\
\text { Practice score }\end{array}$} & Before protocol & $62.29 \pm 2.77$ & $66.92 \pm 9.78$ & $66.02 \pm 8.06$ & 3.50 & $0.038^{*}$ \\
\hline & $\begin{array}{l}\text { Immediately after } \\
\text { protocol }\end{array}$ & $80.56 \pm 6.31$ & $80.04 \pm 5.12$ & $80.85 \pm 5.45$ & 0.06 & $0.93(\mathrm{NS})$ \\
\hline & 3 months after protocol & $77.93 \pm 5.68$ & $77.89 \pm 6.82$ & $81.30 \pm 6.12$ & 0.78 & $0.46(\mathrm{NS})$ \\
\hline & 6 months after protocol & $77.71 \pm 5.77$ & $76.70 \pm 7.54$ & $79.10 \pm 6.91$ & 0.32 & $0.72(\mathrm{NS})$ \\
\hline
\end{tabular}

\section{DISCUSSION :}

Catheter-associated urinary tract infections (CAUTIs) account $40 \%$ of all nosocomial infections in the United States, affecting nearly 900,000 patients per year. (The indwelling urinary catheter (IUC) is a widely utilized device in modern hospital environments, yet they are not always used appropriately in hospital settings and can result in prolonged use and improper management, increasing risk of infections and length of stay. Nurses are the primary champions to promote preventative measures, provide patient education, and evaluate evidence-based practice (EBP) strategies to decrease CAUTIs (Gordon, 2015). Moreover Meddings et al. (2014) stated that changing practice is not easy and can be costly, but it will cost healthcare organizations more financially without adequately educating nurses about best practices. In addition, lack of prevention strategies knowledge regarding patient safety and the quality of care.

Concerning nurses' satisfactory practice total score related to urinary catheterization procedure throughout the protocol intervention. The current study results indicated improvement in the nurses' satisfactory practice total score regarding urinary catheterization procedure throughout the protocol intervention. This finding supported by Damani, (2011) who mentioned that Healthcare personnel performing urinary catheterization should receive training on correct procedures for insertion and maintenance of urinary catheters based on local written protocols. Also Fink et al., (2012) reported that it is critical that staff be made aware of policy revisions and be provided with annual training and education reviews related to catheter insertion and maintenance techniques 
Nurses' satisfactory practice total score related to urinary catheter care throughout the protocol intervention. The results revealed improvement in the nurses' satisfactory practice total score regarding urinary catheter care throughout the protocol intervention. This finding supported by Gordon,(2015) who said that Nurses are required to have the ability to improve patient care, increase positive quality outcomes and decrease negative outcomes with patients.

As regard total score of nurses' practice regarding urinary catheter management throughout the protocol intervention. It revealed that there were statically significant difference in the total score of nurses' practice regarding urinary catheter management throughout the protocol intervention between immediately posttest, pre protocol first follow up and pre protocol, second follow up, and pre protocol. This finding supported by Sobeih and Nasr, (2015) who revealed that, there were highly statistically significant differences between level of acquired nurses' practices pre/post and one month later after the interactive workshop related to urinary catheter insertion, care and removal.

Regarding age of studied nurses the result, of the current study revealed that there were no statistical significance differences between nurse's knowledge or practice and their age groups throughout the protocol intervention. This Finding of this study was consistent with a study conducted by Prasanna and Radhika, (2015) on knowledge regarding catheter care among staff nurses who reported that there is no significant association between the level of knowledge of staff nurses regarding catheter care with their selected socio demographic variables like age, gender and professional experience. The results contradicted with Shahen, (2012) who detected a positive statistically significant relation between post program knowledge score and age of participants

As regard level of studied nurses' experience the results revealed that; there no statically significant differences between studied nurses knowledge and their experience throughout the protocol intervention except before the protocol. In this respect Madeo and Roodhouse, (2009) clarified that nurses who are qualified for at least 4 years, would be expected to have at least a basic understanding of best practice for the management of an indwelling urinary catheter. 


\section{CONCLUSION:}

Based on study findings, it can be concluded that: There were statistically significant difference in the total score of nurses' knowledge as well as practice regarding urinary catheter care throughout the protocol intervention between posttest, pre protocol first follow up and pre, second follow up, and pre protocol. A high statistical significant improvement in nurse's knowledge were found between the immediate posttest and the pre protocol level, between pre protocol and 3month after and between pre-second follow up.

\section{RECOMMENDATIONS:}

Providing ongoing in service education for nurses to update their practice related to care of patients undergoing urinary catheterization .Provide equal opportunities for nurses to attend national and international congresses and in-service training programs related to urinary catheter care and urological departments and other units where catheters are used must be supplied with comprehensive manuals containing basic procedural steps of how to handle and to avoid catheter associated problems.

Developing nursing standards of care for patients with urinary indwelling catheter and encouraging nurses to attend national and international congresses, seminars, symposiums and workshop about urinary catheterization, care of the patient with urinary catheter.

\section{REFERENCES:}

Gordon P.( 2015): The Effects of Nursing Education on Decreasing Catheter Associated Urinary Tract Infection Rates, Doctor of Nursing Practice (DNP), College of Health Sciences, Walden University,P.1-18.

Pui L. (2011): Nurse-led weaning of short-term urinary tract catheters to reduce duration of unnecessary catheterization in acute general ward setting, degree of Master of Nursing at the University of Hong Kong ,P.1-20. 
Yoon B., McIntosh S., Rodriquez L., Holley A., Faselis J., Liappis P. (2013): Changing behavior among nurses to track indwelling urinary catheters in hospitalized patients. Interdisciplinary Perspectives on Infectious Diseases, p.1-4.

Altun, I., Karakoc, A. (2010): The impact of an interactive workshop on the management of urinary catheterization on nurses. Journal of Urologic Nursing; 4(3): $125-132$.

Ercole F, Macieira T, Wenceslau L., Macieira T., Martins A., Campos C. and Chianca T. (2013): Integrative review: evidences on the practice of intermittent/indwelling urinary catheterization. Rev Lat Am Enfermagem 2013; 21:459-68

Oman K S. , Makic MB. , Fink R., Schraeder N., Hulett T., Keech T., Tarah and Wald H. (2012): Nurse-directed interventions to reduce catheter-associated urinary tract infections," American Journal of Infection Control, vol. 40, pp. 548-553, 2012

Meddings J., Rogers, M. M., Krein, S. L., Fakih, M. G., Olmsted, R. N., \& Saint, S. (2014). Reducing unnecessary urinary catheter use and other strategies to prevent catheter-associated urinary tract infection: an integrative review. BMJ Quality \& Safety, 23(4), 277-28289.

Damani N. (2011): prevention of catheter-associated urinary tract. Chapter 18; International Federation of Infection Control (I FIC); 2nd Edition - Revised 2011 (Previously published as Infection Control: Basic Concepts and Training.

Fink R., Gilmartin H., Richard A., Capezuti E., Boltz M. and Wald H. (2012): Indwelling urinary catheter management and catheter-associated urinary tract infection prevention practices in Nurses Improving Care for Health system Elders hospitals. Association for Professionals in Infection Control and Epidemiology ,American Journal of Infection Control P.1-6

Sobeih H. and Nasr M. (2015): Indwelling Urinary Catheter Management: Effect of an Interactive Workshop on Nurses' practice and Perception. New York Science Journal 2015;8(5).PP.117-124. 
Prasanna K., Radhika M. (2015): Knowledge regarding Catheter care among Staff Nurses. International Journal of Applied Research 2015; 1(8): 182-186

Shahin, M., Mohamed, W., and Sayd, M. (2012): Nurses Knowledge and Practices Regarding Enteral Nutrition at the Critical Care Department of Al- Manial University Hospital in Egypt: Impact of a Designed Instructional Program. Journal of American Science, 8 (11). 397- 404.

Madeo M. and Roodhouse AJ. (2009): Reducing the risks associated with urinary catheters. Nursing Standard. Vol (23), 29, p. 47-55. 


\section{تأثير بروتوكول لرعاية المرضى الخاضعين للقسطرة البولية على ممارسات الممرضين}

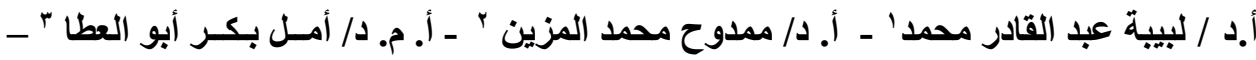

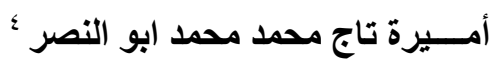

' أستاذ التصريض الباطني الجر/حي ـ كلية التصريض - جامعة القاهرة / أ أستاذ جراحة الاوعية الدموية

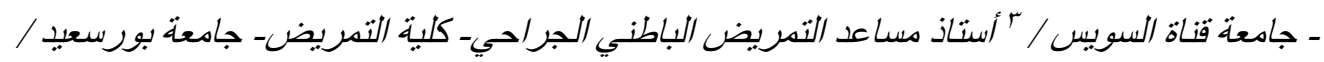

$$
\text { ؛ أخصائي تمريض - جامعة الازهر. }
$$

تعتبر القسطرة البولية واحدة من اهم و أكثر الاجراءات العلاجية المستخدمة في المستشفيات وتثتمل على ادخال انبوب مجوف من خـلال مجرى البول الى المثانـة وذلك لتيسير عملية خروج البول من المثانـة. تمثل القسطرة البولية ضـرورية لنسبة كبيرة من المرضى في المستشفيات وخاصـة الذين يعانون من اضطر ابات في الجهاز

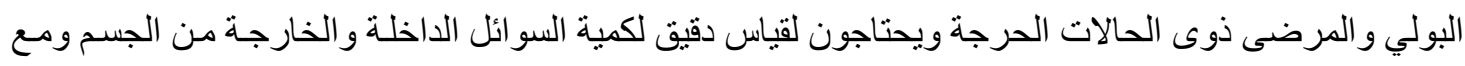
وجود اهمية لاستخدام القسطرة البولية يوجد ايضا العديد من المضاعفات ومن اهمها عدوى مجرى البول وتؤدى الإصابة بعدوى مجرى البول المصاحبة للقسطرة البولية. . وقد أجريت در اسة شبه تجريبية مع تقييم قبلي وبعدى

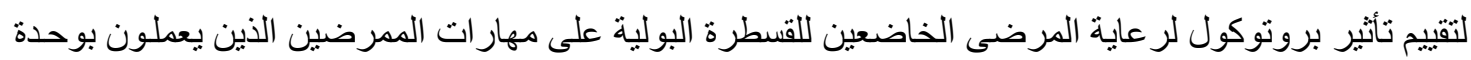
الرعاية المركزة وقسم المسالك البولية وقسم الباطنـة الداخلي بمستشفى جامعة الاز هر بدمياط. وقد شملت عينـة الدر اسـة على جميع الممرضـات و الممرضين الذين يعملون بالأمـاكن السـابق ذكر هـا. وقد تم استخدام استمارة استبيان للبيانات الثخصية واستمارة تقييم الاداء فيما يتعلق بر عايـة المرضى الخاضعين لتركيب القسطرة البولية.

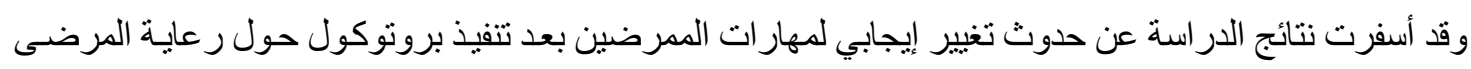
الذين يخضعون للقسطرة البولية وقد أوصت الدر اسة بتَزويد الممرضات بالتدريب المستمر لتحديث مهار اتهم عن بن

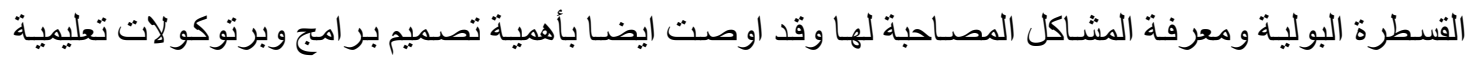
لتحقيق جودة عالية من الرعاية التمريضية للقسطرة البولية.

الكلمات المرثدة: بروتوكول الرعاية ، القسطرة البولية ،ممارسات الممرضين . 